\title{
Systems biology of development and differentiation: Editorial Overview
}

\author{
Philip Greulich $^{1,2}$ and Ben MacArthur ${ }^{1,2,3}$ \\ ${ }^{1}$ Mathematical Sciences, University of Southampton, Southampton SO171BJ, UK \\ ${ }^{2}$ Institute for Life Sciences, University of Southampton, Southampton SO171BJ, UK \\ ${ }^{3}$ Centre for Human Development, Stem Cells and Regeneration, Faculty of Medicine, \\ University of Southampton, Southampton SO171BJ, UK
}

Recent years have seen increasing interest in using systems biology methods to understand the dynamics of development and cellular differentiation. This edition of Current Opinion in Systems Biology focusses on some of the exciting emerging work in this area.

Development and differentiation are complex processes, involving interplay between molecular, cellular, and multicellular regulatory mechanisms. This requires coordination of cell dynamics at multiple different length and time scales. The eight articles in this collection therefore span the range of biological processes involved in regulating cell proliferation, differentiation, and morphogenesis, from the molecular to the organismal scale. These works demonstrate how interdisciplinary approaches that involve mathematical and biophysical modelling may lead to novel insights about these processes.

At the molecular level Babtie et al. address the question of how the intracellular molecular regulatory networks that define cell identities, and guide cell fate changes, can be reliably inferred from gene expression data. They focus particularly on the problem of accurate inference of regulatory networks from the high-throughout single cell expression data that is becoming increasingly generated, and the issues that arise when considering samples undergoing some dynamical process such as differentiation. Inferring genuine functional interactions while filtering spurious associations is a significant statistical problem. To address this issue, Babtie et al. discuss how methods from dynamical systems theory, and recent advances in multivariate information theory are being used to infer more accurate networks and explore the ways that regulatory networks reconfigure over time during differentiation.

In complement to the reverse engineering process described by Babtie et al., Prochazka et al. consider an alternative strategy that uses principles from synthetic biology to "forward engineer" regulatory circuits in stem cells toward defined outputs, and by doing so better understand how simple regulatory modules may be connected to direct complex cell fate decisions. They argue that iteration between forward and reverse engineering strategies will provide a better understanding of the structure of natural genetic regulatory networks and the 
dynamics of the "building blocks" from which they are constructed. This is a rich area, which not only uses notions from engineering, mathematics and biology to better understand cellular dynamics but also presents some exciting engineering, statistical and mathematical challenges.

A central challenge in this area is how best to interpret the cell-cell variability that is inevitably present within cell populations, for example to distinguish functionally relevant variation from technical noise. To address these issues, Miragaia et al., outline how recent experimental and computational methods are helping us to better understand both cellular differentiation at the single cell level and heterogeneity within cellular populations, focussing specifically on T cell development as an example. Again, this is an area of tremendous potential, and significant challenge. Single cell technologies are already able to produce vast amounts of data; in the near future these methods are likely to develop rapidly, and present further analytical challenges.

Moving onto the cellular level, Fachetti et al. consider the question of how cell growth is coordinated within the cell cycle, and how the cell size at division is regulated. While cell cycle transit has been well understood for several decades, this review looks at more recent discoveries about cell size control, using simple unicellular model organisms as an example. Three main principles of cell size control are identified: "sizers" always divide when a cell reaches a given size, "adders" divide when a fixed size increment has been added, and "timers" divide at fixed time intervals. Fachetti et al. explain how these different principles can be determined experimentally and discuss which molecular mechanisms may be involved. They show in particular how synergy between experiments and mathematical modelling can provide new ways to explore the underlying molecular mechanisms for cell size control.

While Fachetti et al. examine general principles of cell division, many somatic cells do not divide, and others exhaust their proliferative potential after a few divisions, exit the cell cycle, and commit to terminal differentiation. Indeed, only stem cells have the ability to selfrenew indefinitely. Stiehl and Marciniak-Czochra discuss the importance of stem cell selfrenewal for tissue development, maintenance and regeneration, and, as the flip side of the coin, in tumour development. They note that self-renewal, when dysregulated to prevail over differentiation, leads to an uncontrolled expansion of the stem cell pool that can drive tumour growth. Interestingly, they point to recent developments that indicate that it may be the propensity of stem cells to divide symmetrically that drives tumour growth, even if divisions are slow, rather than the rate of proliferation per se. Based upon this observation it is 
suggested that self-renewal pathways, rather than fast cycling cells, could be effective targets in tumour treatment.

At the multicellular level, Naganathan and Oates discuss the physical and biochemical factors that are coordinated during tissue morphogenesis. They identify three mutually interacting factors of importance - biochemical signalling, mechanical forces and geometry and explain these mechanisms using four well-studied model organisms. In recent years, it has become evident that mechanical forces not only shape animal embryos, but through mechanosensing, they can be translated into biochemical signals, which eventually instruct cell fate choices during development. Naganathan and Oates discuss recent approaches to the longstanding question of how spatial heterogeneities first arise from an initially uniform cluster of cells (which ultimately become consolidated into complex patterns with different cell types arranged in well-defined spatio-temporal patterns). Several mechanisms for the essential initial "symmetry breaking" are explained, based on the most recent discoveries in developmental biology and biophysics.

In a complementary article Fisher et al. consider in some detail the ways in which chemical and mechanical mechanisms interact during epithelial morphogenesis specifically. Integrating these inherently complex mechanisms, which act on a range of different length and timescales, into a holistic view of morphogenesis is a significant challenge, and a rich research area. Since deciphering this complexity is difficult using intuition and experiment alone, in their review Fisher et al. show how mathematical models can be used in combination with experiment to advance our understanding.

In the final article in this collection, Rulands and Simons discuss how principles from statistical physics can be used to connect the molecular, cellular and multicellular scales. They observe that despite recent progress in unravelling some of the basic genetic "laws" of life, it is evident that knowledge of these principles does not immediately translate into a deeper understanding of how an entire organism unfolds during development on a macroscopic scale. It is the "emergence" of complex macroscopic behaviour from microscopic principles that determines the properties of developing tissues. A quantitative understanding of translation between the scales is therefore paramount to our understanding of development. Rulands and Simons discuss how principles from statistical physics can contribute to this understanding. They point out that historically a major endeavour in statistical physics has been to understand how the microscopic laws of elementary particles unfold to create the large-scale properties of matter, and transitions between different phases of matter (e.g. the transitions between solid and fluid). They argue that similar mathematical 
concepts can help us to understand how molecular dynamics naturally lead to large-scale properties of tissues. They furthermore suggest that general principles such as "universality" and "scaling" may be equally important as specific cellular processes to understand qualitative aspects of development, arguing that the microscopic details of a system are often relatively unimportant in understanding its large-scale properties, which may instead emerge from relatively simple, paradigmatic rules.

As a whole, the collection highlights a number of themes of general importance. Firstly, the articles demonstrate that development emerges from an interplay of cell and tissue dynamics at multiple different length and time scales. This coordination is achieved by information exchange within and between cells by both physical and biochemical mechanisms.

Secondly, to understand these inherently complex processes requires a strongly interdisciplinary approach that includes biologists, physical scientists, engineers, and mathematicians. Experimental methods alone are often not sufficient: mathematical analysis and modelling are essential to advance our understanding and to make quantitative predictions. Indeed, as experimental methods becoming more advanced, and producing larger amounts of more nuanced data, mathematical methods are becoming more and more important.

A particular area, that is highlighted in a number of the reviews in this collection is the ability of mathematical methods and principles from statistical physics help to "bridge the scales" by allowing predictions of macroscopic "emergent" tissue properties from knowledge of microscopic principles, and vice versa. We anticipate that we will see increasing use of methods from mathematics and physics to better understand biological phenomena at the systems level, including those in development and differentiation, in the coming years. 